\title{
Correction for the effect of atmospheric sound absorption on the sound absorption coefficients of materials measured in a reverberation room
}

\author{
Yoshito Hidaka, Hiroo Yano, and Hideki Tachibana \\ Institute of Industrial Science, University of Tokyo, \\ 7-22-1, Roppongi, Minato-ku, Tokyo, 106 Japan
}

(Received 17 September 1987)

\begin{abstract}
When measuring the sound absorption coefficient of materials in a reverberation room, changes of the temperature and relative humidity in the room before and after setting a test specimen can cause considerable errors especially in high frequency bands. In order to investigate the correction method for such kind of error, numerical and experimental investigations were made in this study. At first, the calculation method for the correction was investigated, and then, possible errors due to atmospheric condition change were numerically examined based on the calculation method of the sound intensity attenuation loss of air prescribed in ANSI S1.26-1978. As the experimental investigation, a set of reverberation time measurements were made in a reverberation room, with and without a sound absorption material, under various atmospheric conditions. From those results, the sound absorption coefficients of the material were calculated by considering the effect of atmospheric sound absorption. As a result, it was found that fairly consistent values of the sound absorption coefficient can be obtained by making the correction for atmospheric sound absorption even if the temperature and relative humidity conditions vary greatly.
\end{abstract}

PACS number: $43.55 . \mathrm{Ev}$

\section{INTRODUCTION}

The random incident sound absorption coefficient is one of the most important acoustical characteristics of materials, and it is often used in designing room acoustics and noise control. As a practical measurement of this quantity, ISO $354^{1)}$ and JIS A $1409^{2)}$ specify the method of using a reverberation room. This method is the most reliable at the present time and is widely used. However, some problems remain unsolved in this measuring method, such as the diffusivity in a room, the effect of sound diffraction at the edge of the specimen (so-called edge effect) and so on. ${ }^{4}$ )

The most serious problem among them, especially in high frequency bands, is the effect of atmospheric sound absorption. The change of the atmospheric condition in a reverberation room before and after setting a test specimen leads to noticeable errors in the results of sound absorption coefficient. Therefore, requirements for temperature and relative humidity conditions during the measurement are prescribed in the preceding standards. However, it seems to be rather difficult to keep the atmospheric condition constant in actual measurements.

In this study, possible errors due to temperature and relative humidity condition change were examined numerically, and the possibility of correcting the effect of the atmospheric condition change has been examined by measuring the reverberation time in a reverberation room, with and without a sound absorbing material, under various temperature and relative humidity conditions. 


\section{THEORETICAL INVESTIGATION}

2.1 Correction Method for the Effect of Atmospheric Absorption

The reverberation time in a reverberation room with and without a test specimen $\left(T_{1}\right.$ and $T_{2}$, respectively) is expressed as follows by taking into account the change of the atmospheric condition between the two measurements:

$$
\begin{aligned}
& T_{1}=\frac{55.3}{c_{1}} \frac{V_{0}}{\alpha_{0} S_{0}+4 m_{1} V_{0}} \quad[\mathrm{~s}] \\
& T_{2}=\frac{55.3}{c_{2}} \frac{V_{0}}{\alpha_{0}\left(S_{0}-S\right)+\alpha S+4 m_{2} V_{0}} \quad[\mathrm{~s}]
\end{aligned}
$$

where,

$V_{0}:$ air volume of the reverberation room $\left[\mathrm{m}^{3}\right]$

$S_{0}$ : inner surface area of the room $\left[\mathrm{m}^{2}\right]$

$\alpha_{0}$ : mean sound absorption coefficient of the room

$S:$ area of the test specimen $\left[\mathrm{m}^{2}\right]$

$\alpha$ : sound absorption coefficient of the test specimen

$c_{1}, c_{2}$ : sound velocities under the atmospheric conditions in which $T_{1}$ and $T_{2}$ are measured, respectively $[\mathrm{m} / \mathrm{s}]$

$m_{1}, m_{2}$ : sound intensity attenuation coefficients of air under the atmospheric conditions in which $T_{1}$ and $T_{2}$ are measured, respectively $\left[\mathrm{m}^{-1}\right]$

Under the assumption that the sound absorption coefficient $\alpha$ is independent of the atmospheric condition, it is expressed as follows from Eqs. (1) and (2).

$$
\begin{aligned}
\alpha= & \frac{55.3 V_{0}}{S}\left[\frac{1}{c_{2} T_{2}}-\frac{1}{c_{1} T_{1}}\left(1-\frac{S}{S_{0}}\right)\right] \\
& -\frac{4 V_{0}}{S}\left[m_{2}-m_{1}\left(1-\frac{S}{S_{0}}\right)\right]
\end{aligned}
$$

In almost all cases, $S / S_{0}$ is sufficiently smaller than unity. Consequently, Eq. (3) can be approximated as follows:

$$
\alpha=\frac{55.3 V_{0}}{S}\left(\frac{1}{c_{2} T_{2}}-\frac{1}{c_{1} T_{1}}\right)-\frac{4 V_{0}}{S}\left(m_{2}-m_{1}\right)
$$

When the atmospheric condition is kept constant, the following expression is derived, which is adopted in ISO 354 and JIS A 1409.

$$
\alpha=\frac{55.3 V_{0}}{c S}\left(\frac{1}{T_{2}}-\frac{1}{T_{1}}\right)
$$

where, $c=c_{1}=c_{2}$

In order to validate Eq. (5), requirements for temperature and relative humidity are defined in the standards. However, it is rather difficult to keep the atmospheric condition constant before and after the introduction of the test specimen in many cases. Therefore, it is necessary to examine the applicability and validity of the method of correcting the effect of atmospheric condition change using Eq. (4).

\subsection{Numerical Investigation of the Effect of} Atmospheric Absorption

As the second investigation, the error caused by the atmospheric condition change was examined by calculation.

In the right-hand side of Eq. (4), the change of the first term due to temperature change is sufficiently small compared with the change of the second term. Therefore, the examination was made by considering only the second term.

For the calculation of the sound intensity attenuation coefficient of air $(m)$, the method prescribed in the American National Standard ANSI S1.26-1978 ${ }^{3)}$ was adopted.

Figure 1 shows contour maps of the sound intensity attenuation coefficient on relative humidity $\left(h_{\mathrm{r}}\right)$ - temperature $(t)$ plane for the center frequencies of from 1.6 to $5 \mathrm{kHz}$ in $1 / 3$ octave band. In these figures, a contour interval is $0.00125 \mathrm{~m}^{-1}$, which is equivalent to absolute error of 0.1 in sound absorption coefficient under the condition that $V_{0}$ is $200 \mathrm{~m}^{3}$ and $S$ is $10 \mathrm{~m}^{2}$. In the case where $V_{0}$ and $S$ are different from these values, each contour interval means the absolute error of $0.005 V_{0} / S$ in sound absorption coefficient. Thus, the error caused by the atmospheric condition change can be estimated by plotting the temperature and relative humidity conditions before and after introduction of a test specimen on these maps. As is noticed in these figures, the effect of the atmospheric condition change is serious in low temperature and low humidity region, where the contour lines are close.

\subsection{Tolerance of Atmospheric Condition Change}

As mentioned in Sect. 2.1, the tolerances of atmospheric condition are prescribed in ISO 354 and JIS A 1409. In this section, possible errors of sound absorption coefficient caused by the atmospheric condition changes within the tolerances were examined for the case of $V_{0}=200 \mathrm{~m}^{3}$ and $S=10 \mathrm{~m}^{2}$ by 


\section{Y. HIDAKA et al.: MEASUREMENT OF SOUND ABSORPTION COEFFICIENT}
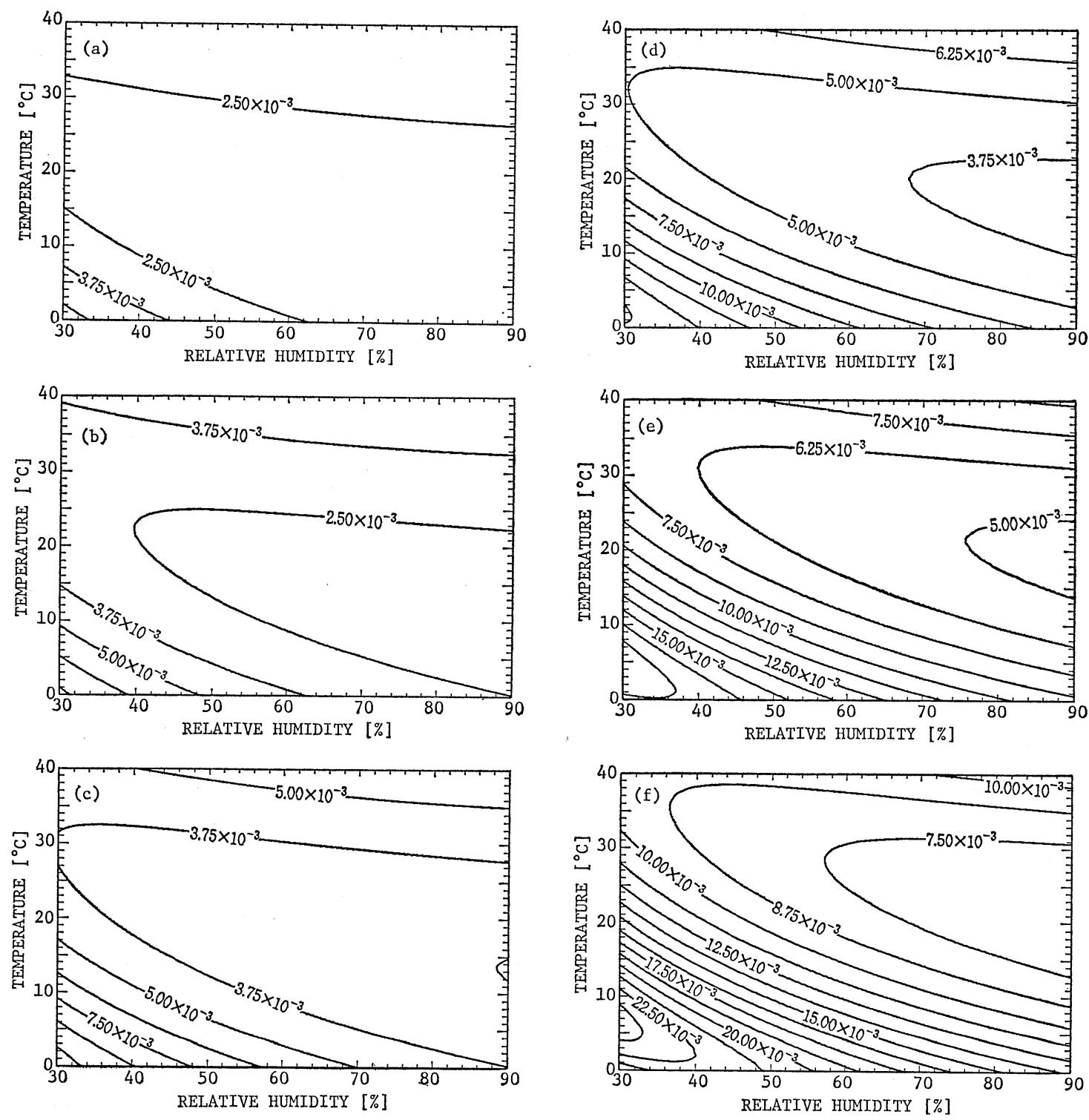

Fig. 1 Sound intensity attenuation coefficient in $\mathrm{m}^{-1}$ for center frequencies in $1 / 3$ octave band. Contour interval is $0.00125 \mathrm{~m}^{-1}$. (a) $1.6 \mathrm{kHz}$, (b) $2 \mathrm{kHz}$, (c) $2.5 \mathrm{kHz}$, (d) 3.15 $\mathrm{kHz}$, (e) $4 \mathrm{kHz}$, (f) $5 \mathrm{kHz}$.

using Fig. 1.

ISO 354 prescribes a lower temperature limit of $10^{\circ} \mathrm{C}$ and a lower relative humidity limit of $40 \%$, and allows the atmospheric condition changes of $3^{\circ} \mathrm{C}, 3 \%$ for $h_{\mathrm{r}}<60 \%$ and $5^{\circ} \mathrm{C}, 5 \%$ for $h_{\mathrm{r}}>60 \%$. In the case of $4 \mathrm{kHz}$, it is found that the maximum error of about 0.2 can arise under the condition near the lower limits of temperature and relative humidity.

In JIS A 1409, a change of temperature less than $5^{\circ} \mathrm{C}$ and that of relative humidity less than $10 \%$ are allowed. These tolerances are much more loose than those in ISO 354. Therefore, large errors can arise under low temperature conditions, even if the changes of atmospheric condition are within the tolerances. For example, in the case of $t=10^{\circ} \mathrm{C}$ and 
$h_{\mathrm{r}}=50 \%$, the error more than 0.3 can truly arise in $4 \mathrm{kHz}$ by the atmospheric condition changes of $5^{\circ} \mathrm{C}$ and $10 \%$.

From the numerical examination above mentioned, it was found that considerably large errors can arise even if the atmospheric condition change is within these tolerances, especially under low temperature and low humidity conditions. Therefore, the tolerances of the atmospheric condition change should be specified much more strictly if the measurement is made according to Eq. (5). However, such strict specification makes the measurement unpractical.

Consequently, the possibility of correcting the effect of atmospheric condition using Eq. (4) and the calculation method of $m$ prescribed by ANSI S1.26 have been examined as mentioned below by making a set of reverberation time measurements, with and without absorbing material, under various atmospheric conditions.

\section{EXPERIMENTAL STUDY}

\subsection{Experimental Method}

Measurements of reverberation time in a reverberation room were carried out from autumn to winter in 1983 under various atmospheric conditions. $^{5,6)}$ The measurements were made in an irregular shaped (pentagonal) reverberation room with a volume of $198 \mathrm{~m}^{3}$ and an inner surface area of $203 \mathrm{~m}^{2}$, in which seventeen vinyl-chloride plate diffusers of each area of $0.9 \mathrm{~m} \times 1.8 \mathrm{~m}$ and $3 \mathrm{~mm}$ thickness were randomly suspended. The humidity was controlled by using a humidifier and a dehumidifier, while the temperature was not artificially controlled. The air in the room was stirred by using a small sized fan before each measurement to ensure a uniform atmospheric condition. The temperature and relative humidity were measured at the center of the room with an Assmann psychrometer. In almost all cases, the temperature change before and after the measurement was less than $1^{\circ} \mathrm{C}$ and the change of relative humidity was less than $3 \%$. The average values before and after the measurement were adopted as the atmospheric conditions for the calculation of the values of $m$.

As a test specimen, glass fiber boards of $50 \mathrm{~mm}$ thickness and $24 \mathrm{~kg} / \mathrm{m}^{3}$ density were laid in total area of $9.9 \mathrm{~m}^{2}$ on the floor of the reverberation room without air space.

The measurement of reverberation time was made by using a computer-controlled measuring system composed of a $1 / 3$ octave band real-time spectrum analyzer (RION SA-25) and a desk-top computer (HP 9816) as shown in Fig. 2. In this system, the sound source signal (broad-band noise) was switched on and off automatically, and the reverberation time in each $1 / 3$ octave band was evaluated from the approximated line over the range of sound pressure level of from $5 \mathrm{~dB}$ to $35 \mathrm{~dB}$ lower than that at the beginning of the decay by using the least squares method. Thus, the reverberation times from $100 \mathrm{~Hz}$ to $5 \mathrm{kHz}$ in $1 / 3$ octave band were obtained simultaneously.

In each measurement, five measurement points were chosen in the room, and the reverberation decay was measured ten times at each point. A series of measurement took about $35 \mathrm{~min}$.

\subsection{Measured Results of Reverberation Time}

From among the data of the reverberation time measured under various atmospheric conditions

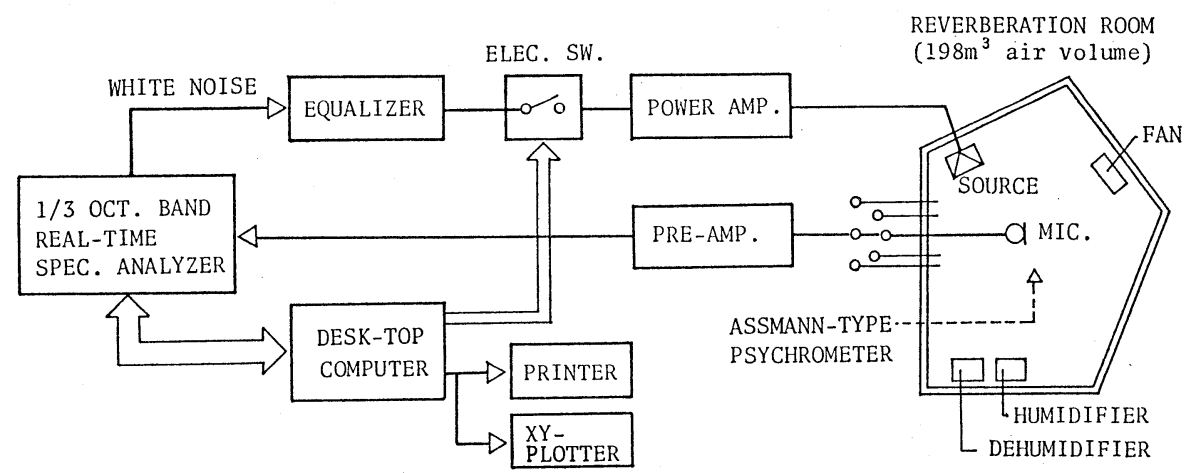

Fig. 2 Measurement system of reverberation time. 


\section{Y. HIDAKA et al.: MEASUREMENT OF SOUND ABSORPTION COEFFICIENT}

Table 1 Atmospheric conditions at the measurements of reverberation time.

\begin{tabular}{cccccc}
\hline \multirow{2}{*}{ Line in figures } & \multicolumn{2}{c}{ Case 1 (Figs. 3 and 4) } & & \multicolumn{2}{c}{ Case 2 (Figs. 7 and 8) } \\
\cline { 2 - 3 } \cline { 5 - 6 } \cline { 5 - 6 } & Empty & With specimen & & Empty & With specimen \\
\hline-- & $15.3^{\circ} \mathrm{C}, 87 \%$ & $15.8^{\circ} \mathrm{C}, 88 \%$ & & $7.3^{\circ} \mathrm{C}, 80 \%$ & $6.9^{\circ} \mathrm{C}, 86 \%$ \\
-- & $15.1^{\circ} \mathrm{C}, 71 \%$ & $15.2^{\circ} \mathrm{C}, 70 \%$ & & $7.0^{\circ} \mathrm{C}, 71 \%$ & $6.8^{\circ} \mathrm{C}, 69 \%$ \\
- & $15.5^{\circ} \mathrm{C}, 61 \%$ & $17.5^{\circ} \mathrm{C}, 60 \%$ & & $6.1^{\circ} \mathrm{C}, 58 \%$ & $6.7^{\circ} \mathrm{C}, 58 \%$ \\
- & $18.0^{\circ} \mathrm{C}, 50 \%$ & $14.0^{\circ} \mathrm{C}, 54 \%$ & & $7.0^{\circ} \mathrm{C}, 50 \%$ & $6.6^{\circ} \mathrm{C}, 53 \%$ \\
- & $17.6^{\circ} \mathrm{C}, 46 \%$ & $16.0^{\circ} \mathrm{C}, 47 \%$ & & $6.9^{\circ} \mathrm{C}, 42 \%$ & $5.1^{\circ} \mathrm{C}, 44 \%$ \\
\hline
\end{tabular}

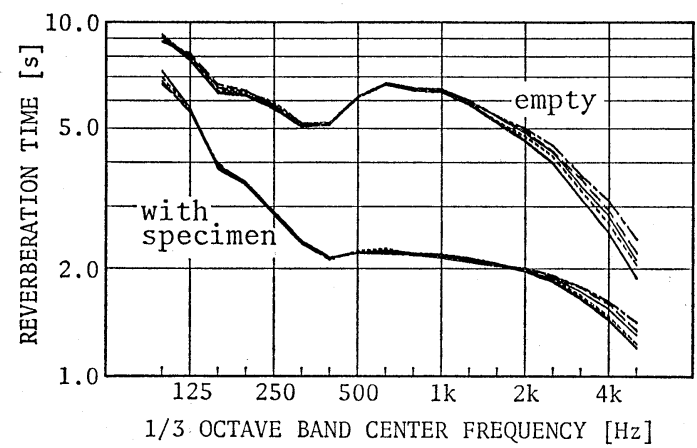

Fig. 3 Reverberation times measured under the conditions of about $16^{\circ} \mathrm{C}$ and relative humidity between 46 to $88 \%$ (case 1 ).

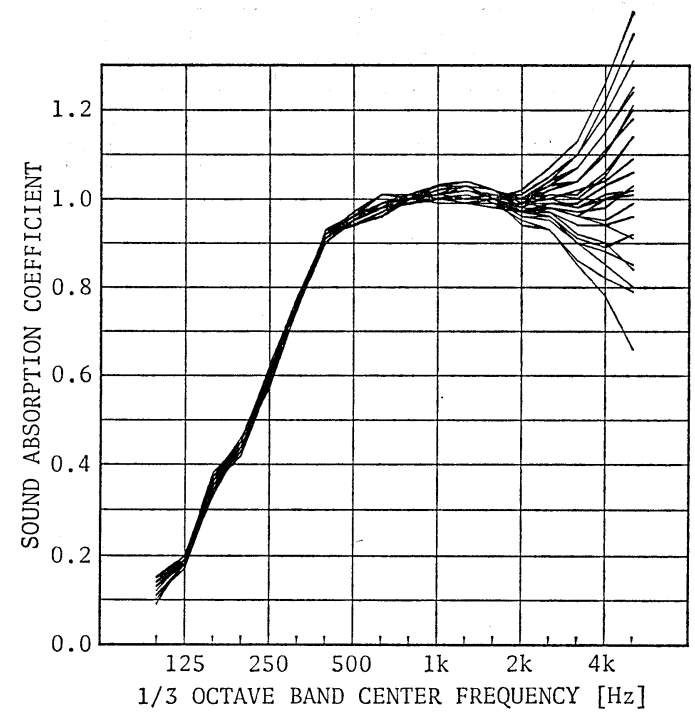

Fig. 5 Sound absorption coefficients of the specimen calculated by disregarding the changes of atmospheric condition (case 1).

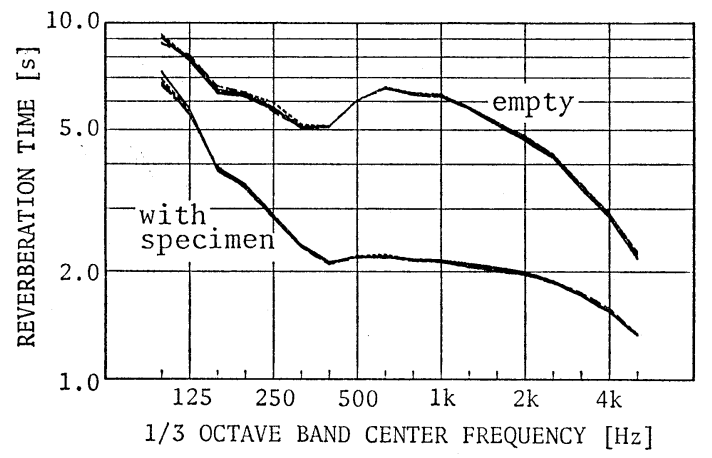

Fig. 4 Reverberation times converted from the values in Fig. 3 into the condition of $20^{\circ} \mathrm{C}$ temperature and $60 \%$ relative humidity (case 1).

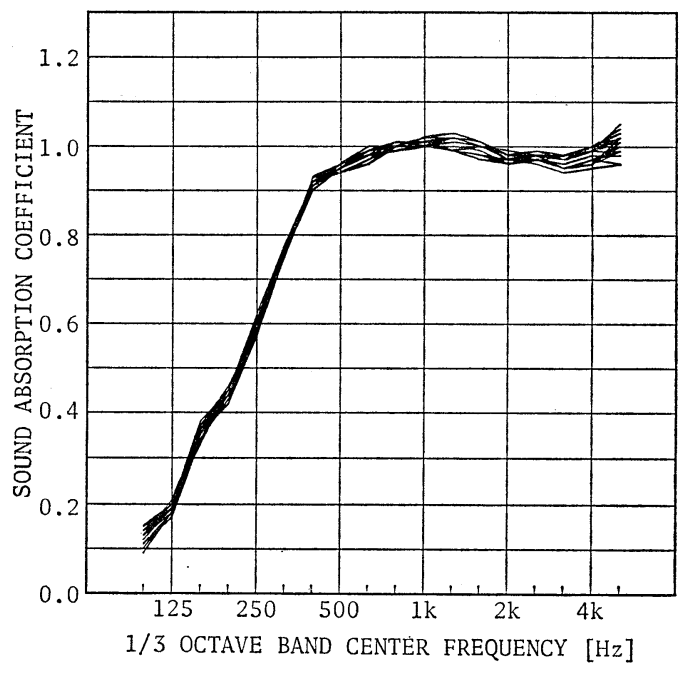

Fig. 6 Sound absorption coefficients of the specimen corrected for the changes of atmospheric condition (case 1). 


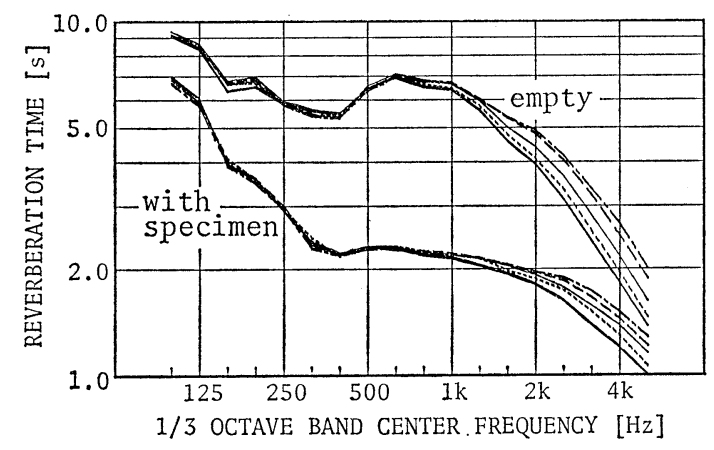

Fig. 7 Reverberation times measured under the conditions of about $6^{\circ} \mathrm{C}$ and relative humidity between 42 to $86 \%$ (case 2 ).

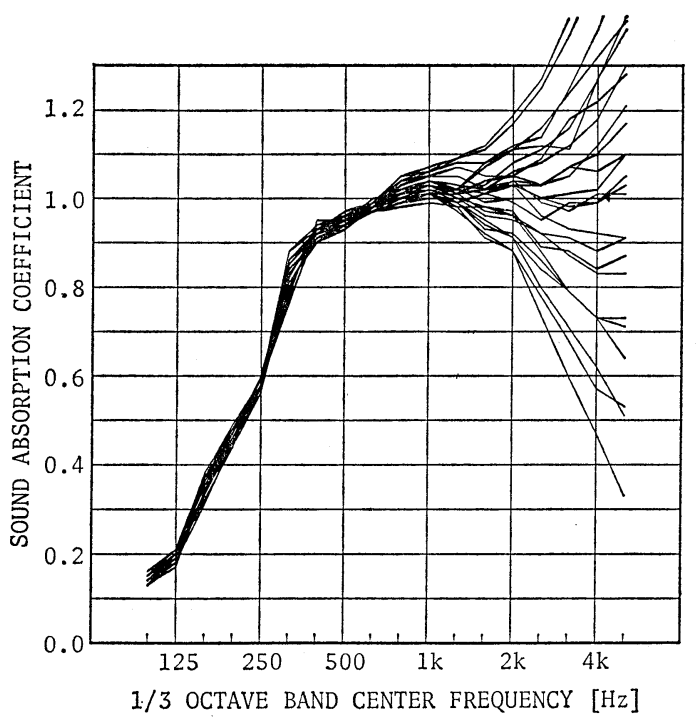

Fig. 9 Sound absorption coefficients of the specimen calculated by disregarding the changes of atmospheric condition (case 2).

with and without the specimen, two groups are shown in Fig. 3 and Fig. 7 (see Table 1). The former data was obtained under relatively high temperature conditions (about $16^{\circ} \mathrm{C}$ ), and the latter under relatively low temperature conditions (about $6^{\circ} \mathrm{C}$ ). As shown in these figures, the change of the reverberation time due to atmospheric condition change is serious in the frequency bands above $1 \mathrm{kHz}$.

Previous to the examination of the sound absorption coefficients, the measured reverberation times were converted into values under the condition of $20^{\circ} \mathrm{C}$ temperature and $60 \%$ relative humidity ac-

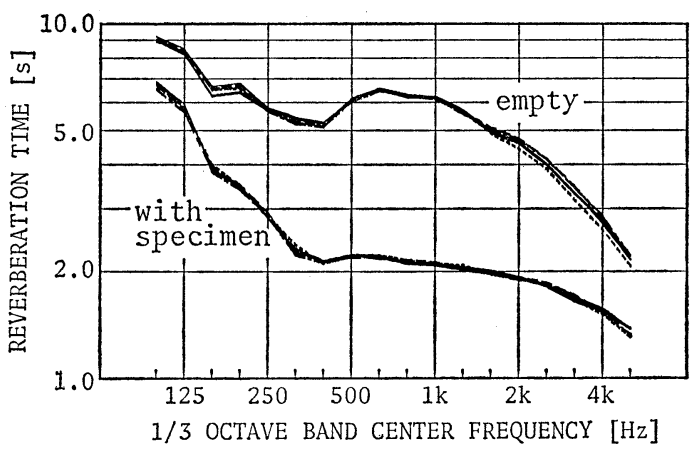

Fig. 8 Reverberation times converted from the values in Fig. 7 into the condition of $20{ }^{\circ} \mathrm{C}$ temperature and $60 \%$ relative humidity (case 2 ).

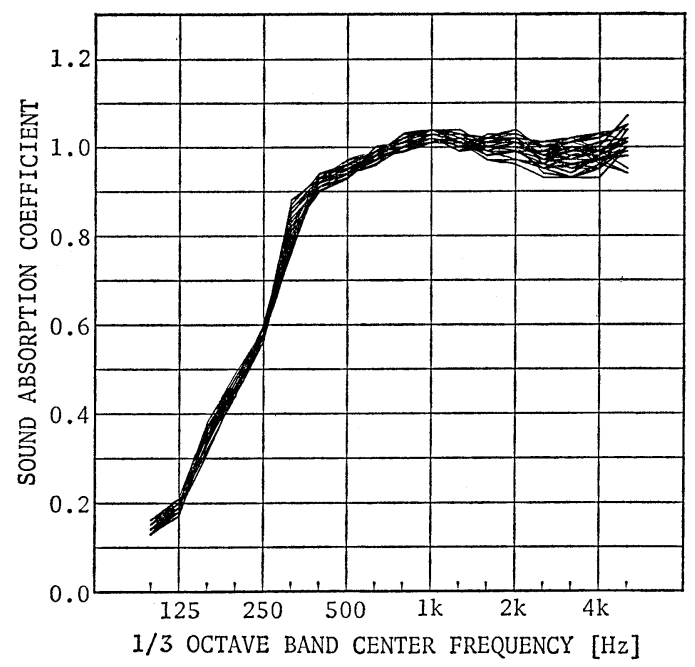

Fig. 10 Sound absorption coefficients of the specimen corrected for the changes of atmospheric condition (case 2).

cording to the following equation which is derived from Eqs. (1) and (2).

$$
T_{\mathrm{c}}=\frac{0.161}{\frac{55.3}{c T}+4\left(m_{\mathrm{c}}-m\right)}
$$

where,

$T$ : measured reverberation time [s]

$T_{\mathrm{c}}$ : reverberation time under the conditions of $20^{\circ} \mathrm{C}, 60 \%$ [s]

$c$ : sound velocity under the atmospheric conditions in which $T$ is measured $[\mathrm{m} / \mathrm{s}]$ 


\section{Y. HIDAKA et al.: MEASUREMENT OF SOUND ABSORPTION COEFFICIENT}

$m$ : sound intensity attenuation coefficient of air during the measurement $\left[\mathrm{m}^{-1}\right]$

$m_{\mathrm{c}}$ : sound intensity attenuation coefficient of air under the condition of $20^{\circ} \mathrm{C}, 60 \%\left[\mathrm{~m}^{-1}\right]$

As a result, the values of the reverberation time have well converged after the correction as shown in Fig. 4 and Fig. 8 .

\subsection{Correction of Sound Absorption Coefficient}

In order to examine the variations of the sound absorption coefficient caused by the atmospheric condition change, the sound absorption coefficients of the specimen were first calculated according to Eq. (5), by randomly combining the reverberation times measured with and without the specimen shown in Fig. 3 and Fig. 7. The result shows great variations especially in high frequency bands as shown in Fig. 5 and Fig. 9.

Secondly, the sound absorption coefficients were calculated according to Eq. (4) which includes the correction for the temperature and relative humidity changes. (In another way, they can also be obtained from the converted reverberation times mentioned above and by using Eq. (5).)

As shown in Fig. 6 and Fig. 10, the sound absorption coefficients have much converged within a deviation of \pm 0.05 after the correction, although extreme relative humdity changes such as $42 \%$ to $86 \%$ was included. This value can be considered sufficiently small when considering the practical use of sound absorption coefficient and its uncertainties caused by other factors.

Judging from these results, it can be expected that the effect of the atmospheric condition change can be sufficiently corrected by the calculation according to Eq. (4) using the ANSI method.

\section{CONCLUSIONS}

A study to improve the accuracy of the sound absorption coefficient measurement by the reverberation room method was made.

Firstly, numerical investigations were made concerning the possible errors due to atmospheric condition change, and the tolerances specified in ISO 354 and JIS A 1409 were examined. As a result, it has been found that noticeable errors can arise even if the changes of temperature and/or relative humidity are within the tolerances.

Secondly, the possibility of correcting the effect of the atmospheric condition change has been investigated by making a lot of measurements of sound absorption coefficient under various atmospheric conditions. As a result, it has been found that even if the temperature and/or relative humidity conditions in a reverberation room change to great extent before and after the introduction of a test specimen, the effect can be sufficiently corrected by using Eq. (4) and the calculation method of the sound intensity attenuation coefficient of air prescribed in ANSI S1.26-1978. (Though this standard is now under revision, it is supposed that the change of calculated sound intensity attenuation coefficient of air is negligibly small for this kind of correction.)

In order to make such a correction effective, it is necessary to realize a uniform atmospheric condition in the reverberation room and to accurately determine the temperature and relative humidity.

\section{REFERENCES}

1) ISO 354, "Measurement of sound absorption in a reverberation room" (1985).

2) JIS A 1409, "Method for measurement of sound absorption coefficients in a reverberation room" (1977) (in Japanese).

3) ANSI S1.26-1978 (ASA 23-1978), "Method for the calculation of the absorption of sound by the atmosphere"' (1978).

4) Y. Makita, M. Koyasu, M. Nagata, and S. Kimura, "Investigations into the precision of measurement of sound absorption coefficients in a reverberation room (I, II)," J. Acoust. Soc. Jpn. (J) 24, 381-402 (1968) (in Japanese).

5) Y. Hidaka, K. Yoshihisa, and H. Tachibana, "On the correction for sound absorption by air in the measurement of reverberant sound absorption coefficient," Proc. Spring Meet. Acoust. Soc. Jpn. 2-6-17, 547-548 (1984) (in Japanese).

6) H. Tachibana, K. Yoshihisa, Y. Hidaka, and K. Ishii, "Correction method for the effect of air absorption on sound absorption coefficients measured in reverberation rooms," Proc. Inter-Noise 84, 1225-1228 (1984). 